\title{
RELATION ENTRE \\ INDICATEUR D'ABONDANCE DE TYPE CPUE \\ ET ESTIMATION DE DENSITÉ PAR ENLĖVEMENTS SUCCESSIFS \\ POUR LES JUVÉNILES DE SAUMON ATLANTIQUE (SALMO SALAR L.) \\ DE L'ANNÉE.
}

\author{
E. PRÉVOST (1) et A. NIHOUARN (2)
}

(1) Laboratoire d'Écologie Aquatique, Institut National de la Recherche Agronomique, 65 rue de St-Brieuc, 35042 Rennes Cedex, France.

(2) Délégation Régionale Bretagne - Basse Normandie, Conseil Supérieur de la Pêche, 84 rue de Rennes, 35510 Cesson Sévigné, France.

\section{RÉSUMÉ}

En rivière, pour estimer ponctuellement sur une station l'abondance d'une population de poissons, la pêche électrique par enlèvements successifs est la méthode la plus couramment utilisée. Pour les $0+$ automnaux de saumon atlantique (Salmo salar) dans les rivières du Massif Armoricain, une technique alternative plus "légère "a été proposée, tout en conservant comme mode de capture la pêche électrique. Elle repose sur un protocole standardisé et fournit des indicateurs d'abondance au niveau stationnel de type capture par unité d'effort (nombre d'individus capturés pour une unité d'effort standard de cinq minutes). Pour bâtir une relation entre ces indicateurs d'abondance et les estimations de densité par enlèvements successifs, les deux types de données ont été recueillis successivement sur les mêmes stations entre 1992 et 1997. Les 44 couples de données collectés montrent qu'il existe une liaison forte entre indicateurs d'abondance de type capture par unité d'effort et estimations de densité $(r=0,88)$, que l'on peut aisément modéliser par une relation directe de proportionnalité :

estimation de densité (ind/100 $\mathrm{m}^{2}$ ) $=\mathrm{A} \times$ indicateur d'abondance (ind/5 mn)

$$
A=0,358 \text {; intervalle de confiance à } 95 \%[0,286 ; 0,430] \text {. }
$$

Cette relation fournit avant tout une équation de conversion des indicateurs d'abondance en densité par $100 \mathrm{~m}^{2}$, même si elle constitue également un élément de validation mutuelle des deux méthodes de quantification de l'abondance des tacons $0+$ automnaux. Les résultats présentés autorisent à spéculer a priori que, pour les juvéniles de saumon $0+$ automnaux et dans des contextes écologiques relativement homogènes, la mise au point d'un protocole de pêche électrique standardisé permet d'obtenir simplement des indicateurs d'abondance proportionnels à des densités estimées par enlèvements successifs.

Mots-clés : indicateur d'abondance, densité, pêche électrique, juvénile, saumon atlantique. 


\section{RELATIONSHIP BETWEEN \\ ABUNDANCE INDICES OF CPUE TYPE AND DENSITY ESTIMATES BY THE REMOVAL METHOD FOR ATLANTIC SALMON (SALMO SALAR L.) YOUNG OF THE YEAR.}

\section{ABSTRACT}

In rivers, successive removals by electrofishing is the most commonly used method for providing local estimates of population abundance at the station level. For Atlantic salmon (Salmo salar) young of the year (YOY) in autumn, a simpler alternative technique has been proposed for the rivers of the Armorican Massif, while still relying on electrofishing for fish capture. It is based on a standardised protocol and gives abundance indices of CPUE type (number of individuals caught for one standard unit of effort of five minutes). In order to establish a relation between these abundance indices and the removal density estimates, the two types of data were gathered successively on the same stations between 1992 and 1997. The 44 data points available show a strong relationship between abundance indices of CPUE type and density estimates $(r=0.88)$, which can be easily modelled by an equation of direct proportionality :

$$
\begin{aligned}
& \text { density estimate }\left(\text { ind } / 100 \mathrm{~m}^{2}\right)=A \times \text { abundance index }(\mathrm{ind} / 5 \mathrm{mn}) \\
& \qquad A=0.358 ; 95 \% \text { confidence interval }[0.286 ; 0.430]
\end{aligned}
$$

This relation provides in the first place a way of converting abundance indices into density per $100 \mathrm{~m}^{2}$, even though it is also an element of cross-validation of the two methods of quantifying YOY salmon abundance. The results presented strongly support that, for YOY salmon in autumn and in relatively homogenous ecological contexts, a standardised protocol of electrofishing allows to obtain easily abundance indices proportional to removal density estimates.

Key-words : abundance index, density, electrofishing, juvenile, Atlantic salmon.

\section{INTRODUCTION}

En rivière, pour estimer ponctuellement sur une station l'abondance d'une population de poissons, la pêche électrique par enlèvements successifs (COWX, 1983) est la méthode la plus couramment utilisée. En dehors des problèmes de biais des estimations obtenues (BOHLIN et COWX, 1990 ; RODGERS et al., 1992 ; RILEY et FAUSCH, 1992 ; RILEY et al., 1993), la méthode par enlèvements successifs présente l'inconvénient d'être coûteuse en temps, en matériel et en personnel. Or, pour comparer des niveaux d'abondance dans l'espace ou dans le temps, estimer la taille d'une population à l'échelle du cours d'eau ou étudier sa distribution spatiale, le nombre de points échantillonnés est généralement le premier déterminant de la validité des résultats (HANKIN, 1984 ; BOHLIN et al., 1990 ; JONES et STOCKWELL, 1995). Ainsi, tout en conservant comme mode de capture la pêche électrique, de nombreux auteurs ont souligné l'intérêt de techniques plus "légères " et ont proposé des alternatives à la méthode classique par enièvements successifs (NELVA et al., 1979 ; STRANGE et al., 1989 ; COBLE, 1992 ; LOBÓN-CERVIÁ et UTRILLA, 1993 ; JONES et STOCKWELL, 1995 ; SIMONSON et LYONS, 1995 ; EDWARDS et al., 1997).

En s'inspirant directement de la technique mise au point par CROZIER et KENNEDY (1994) et en l'adaptant au contexte des rivières du Massif Armoricain, PRÉVOST et BAGLINIĖRE (1995) ont présenté une méthode ciblant sur les 0+ 
automnaux de saumon atlantique (Salmo salan). Elle repose sur un protocole de pêche électrique standardisé et fournit des indicateurs d'abondance au niveau stationnel de type capture par unité d'effort. Se basant sur des arguments théoriques et/ou tirés de la bibliographie, ainsi que sur des essais montrant la bonne répétabilité des mesures réalisées, PRÉVOST et BAGLINIĖRE (1995) justifient la pertinence de leur méthode tout en soulignant l'intérêt d'une mise en relation des indicateurs qu'ils obtiennent avec les estimations de densité déduites de la méthode par enlèvements successifs. Cette mise en relation est le sujet du présent article. Son objet est double :

- fournir un élément de validation mutuelle des deux méthodes ;

- proposer une relation de conversion des indicateurs d'abondance de type capture par unité d'effort en densité de population par unité de surface pour assurer une continuité des séries d'observations collectées depuis plusieurs décennies sur les densités de juvéniles de saumon en Bretagne et en Basse Normandie.

\section{MATÉRIEL ET MÉTHODES}

Entre 1992 et 1997, 44 couples de données (indicateur d'abondance de type CPUE ; estimation de densité par enlèvements successifs) ont été recueillis. Le laps de temps séparant la mise en cuvre successive des deux types de technique sur une même station est en moyenne de 14 jours et varie de 3 jours à un peu plus d'un mois (39 jours). Dans 23 cas, l'indicateur d'abondance a été récolté en premier, la situation inverse étant intervenue dans 21 cas. Les stations correspondent à des habitats préférentiels des juvéniles d'âge 0+ à cette période de l'année (courants, peu profonds et avec un substrat grossier), c'est-à-dire essentiellement des faciès d'écoulement turbulents de type radier ou rapide selon la classification de BAGLINIĖRE et CHAMPIGNEULLE (1982), voire de type “plat courant " (fast run) tel que décrit par BAGLINIĖRE et al. (1993). Les zones prospectées sur chaque station ne sont pas à chaque fois rigoureusement identiques pour les deux techniques utilisées, mais elles se recouvrent toujours au moins partiellement. La largeur des cours d'eau au niveau des stations échantillonnées varie de 3 à 17 mètres (moyenne : $7,6 \mathrm{~m}$ ). Quelle que soit la mesure d'abondance concernée, tous les juvéniles de saumon capturés ont été mesurés (longueur fourche, $\mathrm{mm}$ ) pour chaque opération de pẽche électrique. Les tacons de l'année ont été distingués de leurs congénères plus âgés sur un simple critère de taille après examen de la distribution de la longueur fourche des juvéniles capturés. Pour lever certains cas douteux, il a aussi été procédé à quelques prélèvements d'écailles, bien qu'au niveau d'une même station les cas de recouvrement des gammes de taille des $0_{+}$avec celles des classes d'âge supérieures (essentiellement $1+$ ) soient très rares.

Les indicateurs d'abondance ont été recueillis selon le protocole standardisé décrit par PRÉVOST et BAGLINIĖRE (1995). Sans reprendre en détail la description faite par ces auteurs, on rappellera cependant que la standardisation porte sur :

- le matériel utilisé, à savoir l'appareil portatif de pêche électrique (modèle "Martin pêcheur", constructeur Dream electronic) et les épuisettes, toujours rigoureusement du même type ;

- l'équipe de pêche, composée de quatre personnes : une pour le générateur de courant électrique et l'anode, deux pour les épuisettes et une récupérant les poissons capturés ;

- la puissance électrique, maintenue aux alentours de $50 \%$ de la puissance maximale de l'appareil ; 
- le temps de pêche, cinq minutes de temps effectif, c'est-à-dire durant lequel le courant électrique passe dans l'eau ;

- le type d'habitat physique prospecté, correspondant aux preferundums des jeunes saumons de l'année en fin d'été début d'automne (cf. supra);

- le type de poissons recherchés, à savoir une seule espèce (saumon atlantique) et un seul stade (tacon de l'année) associé à une gamme de taille réduite (6 à $12 \mathrm{~cm}$ en fin d'été début d'automne) ;

- la procédure de pêche elle-même, qui consiste en une succession de balayages de l'anode sur quelques mètres dans le sens du courant, distribuée en progressant latéralement et vers l'amont, en veillant à ne pas soumettre plus d'une fois une même zone au champ électrique. A chaque balayage, on tente de capturer le maximum de jeunes saumons attirés ou « choqués " dans le voisinage immédiat de l'anode, soit en les guidant avec cette dernière en direction des deux épuisettes maintenues fixes sur le fond et filtrant la veine d'eau soumise au courant électrique, soit en les récupérant au moyen d'une petite épuisette mobile.

Cette standardisation du protocole vise à assurer lors de chaque opération un effort de pêche et une capturabilité (i.e. probabilité de capture des poissons soumis à une unité d'effort de pêche) aussi constants que possible. Les indicateurs d'abondance recueillis s'expriment directement par le nombre d'individus capturés pour une unité d'effort de cinq minutes de pêche (ind/5 mn) selon le protocole standardisé. On ne peut associer directement à ces mesures d'abondance des variances d'estimation. Néanmoins, on retiendra que PRÉVOST et BAGLINIĖRE (1995) ont observé des écarts relatifs faibles entre répliquats sur une même station (inférieurs à $13 \%$ ).

Les estimations de densité ont été obtenues à partir d'opérations de pêche électrique menées selon la pratique couramment utilisée en Bretagne et en Basse Normandie, à savoir deux passages successifs sur des secteurs non délimités par des filets, le nombre d'anodes (une à quatre) étant ajusté selon la largeur du cours d'eau. Les surfaces prospectées varient de $172 \mathrm{~m}^{2}$ et $1599 \mathrm{~m}^{2}$ (moyenne : $699 \mathrm{~m}^{2}$ ). En s'inspirant de la méthode proposée par CARLE et STRUB (1978), les estimations d'effectifs ont été réalisées selon une approche bayesienne retenant l'hypothèse d'une probabilité de capture (efficacité de pêche) constante au cours des passages successifs quel que soit l'individu. La taille de la population $(N)$ et l'efficacité de pêche $(p)$ sont estimées par le couple de valeurs le plus probable de leur distribution conjointe a posteriori connaissant les effectifs capturés à chaque passage. Cette distribution de probabilité a posteriori est obtenue :

- en calculant la vraisemblance conjointe de $N$ et $p$ selon un modèle binomial tel que présenté par MORAN (1951, in SCHNUTE, 1983) ;

- en retenant des distributions de probabilité a priori indépendantes pour $N$ et $p$ suivant des lois uniformes comprises respectivement entre l'effectif pêché et cinq fois cette valeur et entre 0,2 et 1 .

Les bornes supérieures pour $N$ et inférieures pour $p$ retenues intègrent le fait que les jeunes saumons sont, en eau courante peu profonde, aisément capturables par pêche électrique (Institut National de la Recherche Agronomique et Conseil Supérieur de la Pêche, données non publiées). Cette information introduite dans le modèle par l'intermédiaire des distributions a priori a pour effet de limiter l'amplitude de la distribution a posteriori de $N$ dans les cas où les effectifs pêchés sont très faibles (quelques unités). On considère ainsi que, pour une espèce comme le saumon, pour laquelle la pêche électrique est très efficace, la configuration où l'expérience de prélèvements successifs capture à chaque passage une très faible proportion d'une population de très grande taille est impossible (voir SCHNUTE (1983) pour plus de détails sur ce point). 
Les intervalles a posteriori à $95 \%$ de $N$ sont déduits de sa distribution marginale a posteriori. Les estimations de densité et leurs limites a posteriori à $95 \%$ sont obtenues en divisant les effectifs estimés et leurs limites a posteriori à $95 \%$ par la surface des secteurs prospectés ; elles sont exprimées en nombre d'individus par $100 \mathrm{~m}^{2}$ (ind/100 $\mathrm{m}^{2}$ ).

L'analyse de la relation entre indicateur d'abondance et estimation de densité a été menée dans le cadre d'un modèle linéaire simple avec l'indicateur d'abondance comme variable indépendante et l'estimation de densité comme variable dépendante, en utilisant pour l'ajustement la méthode des moindres carrés. Pour les intervalles de confiance à $95 \%$ et les tests usuels (au seuil de $5 \%$ ) sur les paramètres du modèle, il a été procédé à des rééchantilionnages "bootstrap" (2000 réalisations). Les distributions des estimateurs ont été approchées par celles déduites directement des échantillons " bootstrap ". Les résidus des estimations de densité obtenues par ajustement linéaire ont été analysés. La nature de leur distribution a été examinée par un test de Komolgorov-Smirnov. Leur dépendance (linéaire) par rapport à cinq variables annexes (superficie et largeur de la station, écart entre les dates des deux types d'opérations, taille moyenne des individus capturés, écart-type des estimations de densité) a été évaluée. L'ensemble des analyses a été réalisé grâce au logiciel SYSTAT (ANONYME, 1996a).

\section{RÉSULTATS}

La précision des estimations de densité de juvéniles de saumon d'âge $0+$ utilisées, appréciée par l'amplitude de leurs intervalles a posteriori à $95 \%$, est inférieure à 5 ind $/ 100 \mathrm{~m}^{2}$ dans $75 \%$ des cas (Figure 1). Seules trois données de densité sont associées à des intervalles a posteriori à $95 \%$ d'amplitude supérieure à $10 \mathrm{ind} / 100 \mathrm{~m}^{2}$. En règle générale, les estimations de densité sont proches des limites basses de leurs intervalles a posteriori à $95 \%$. Ceci est dû à de faibles nombres d'individus capturés lors des pêches électriques par enlèvements successifs (moins de 50 dans $60 \%$ des cas) et/ou à de faibles efficacités de pêche (inférieures à $70 \%$ dans $23 \%$ des cas).

Les observations couvrent une large gamme, allant de 0 à 52 ind $/ 100 \mathrm{~m}^{2}$ pour les estimations de densité et de 0 à 110 ind $/ 5 \mathrm{mn}$ pour les indicateurs d'abondance (Figure 1 ). Néanmoins, quel que soit le type de mesure considéré, les données ont une distribution dissymétrique concentrée du côté des faibles valeurs : les médianes sont à 5 ind $/ 100 \mathrm{~m}^{2}$ pour les densités estimées et $20 \mathrm{ind} / 5 \mathrm{mn}$ pour les indicateurs d'abondance et seulement $25 \%$ des valeurs sont supérieures à $14 \mathrm{ind} / 100 \mathrm{~m}^{2}$ pour les estimations de densité et à $39 \mathrm{ind} / 5 \mathrm{mn}$ pour les indicateurs d'abondance.

L'ajustement d'une relation linéaire entre les indicateurs d'abondance et les estimations de densité (Figure 2) permet d'expliquer $78 \%$ de la variabilité de ces dernières (coefficient de corrélation : 0,88 ). L'ordonnée à l'origine associée à cette relation $(0,290)$ est proche de 0 . La distribution bootstrap de ce paramètre donne un intervalle de confiance à $95 \%$ englobant la valeur nulle $[-1,431 ; 2,311]$. Un modèle de proportionnalité directe entre indicateur d'abondance et estimation de densité a donc été secondairement testé. Son ajustement aux observations est de la même qualité que le précédent, la somme des carrés des écarts passant de 1209 à 1211 . L'essentiel (67\%) de cette variabilité résiduelle est dû à seulement quatre couples d'observations (Figure 2, points encadrés). La pente de la droite de proportionnalité $(0,358)$ est significativement différente de 0 , son intervalle de confiance bootstrap excluant la valeur nulle $[0,286 ; 0,430]$. La distribution des résidus d'estimatians autour de cette droite ne s'écarte pas significativement (au seuil de $5 \%$ ) d'une distribution normale centrée sur 0 . Aucune des variables annexes testées (superficie et largeur de la station, écart entre les dates des deux types d'opérations, taille moyenne des individus capturés, écart-type des estimations de densité) ne permet d'expliquer les résidus $\left(R^{2}<0,1\right)$. 

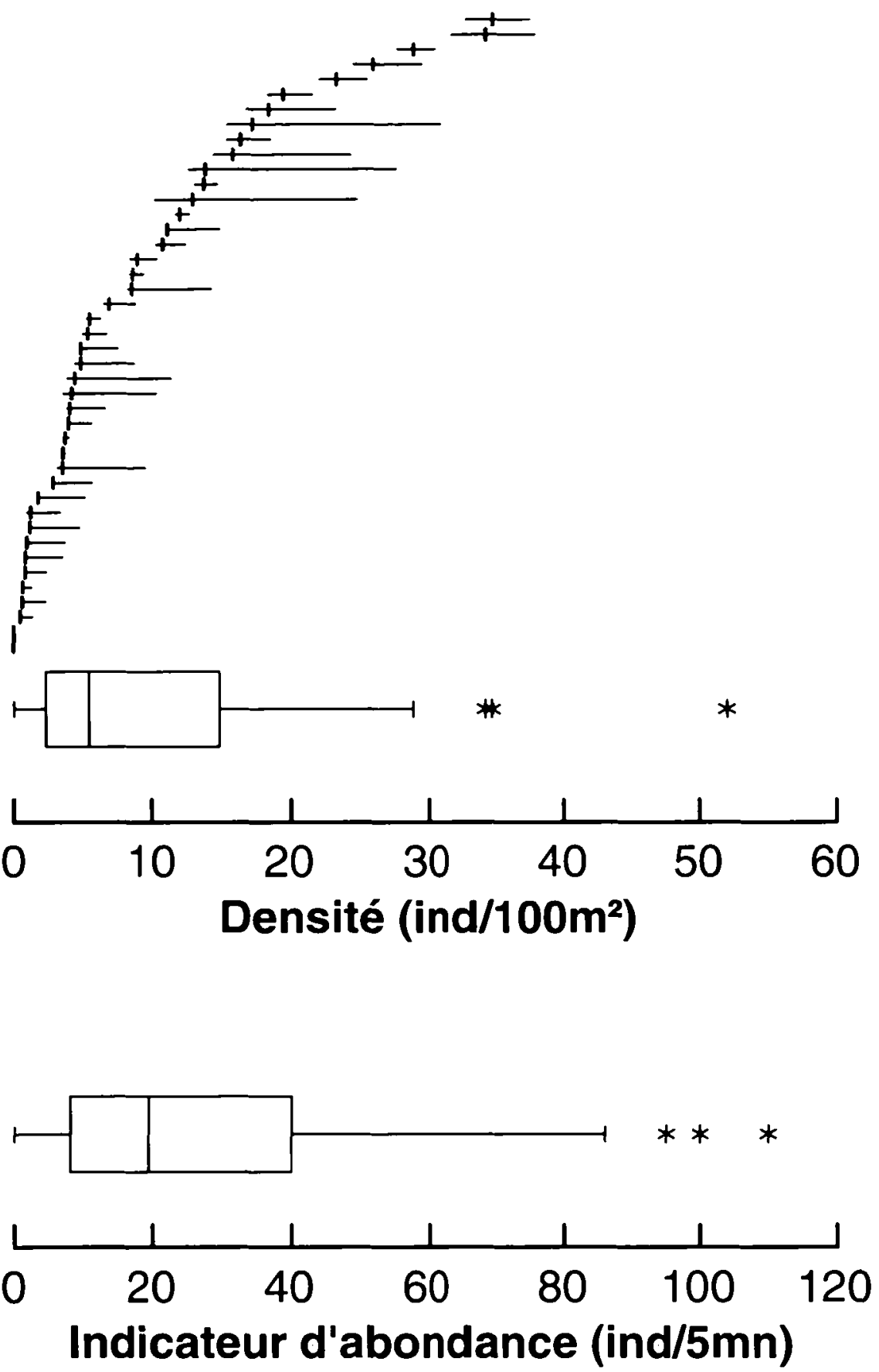

\section{Figure 1}

Boîtes de dispersion (ANONYME, 1996b) des indicateurs d'abondance et des estimations de densité (voir Matériel et méthodes). Pour ces dernières, leurs intervalles a posteriori à $95 \%$ sont également indiqués.

\section{Figure 1}

Box plots (ANONYME, 1996b) of abundance indices and density estimates (see Matériel et méthodes). For the latter, the $95 \%$ posterior intervals are also indicated. 


\section{Densité (ind/100m²)}

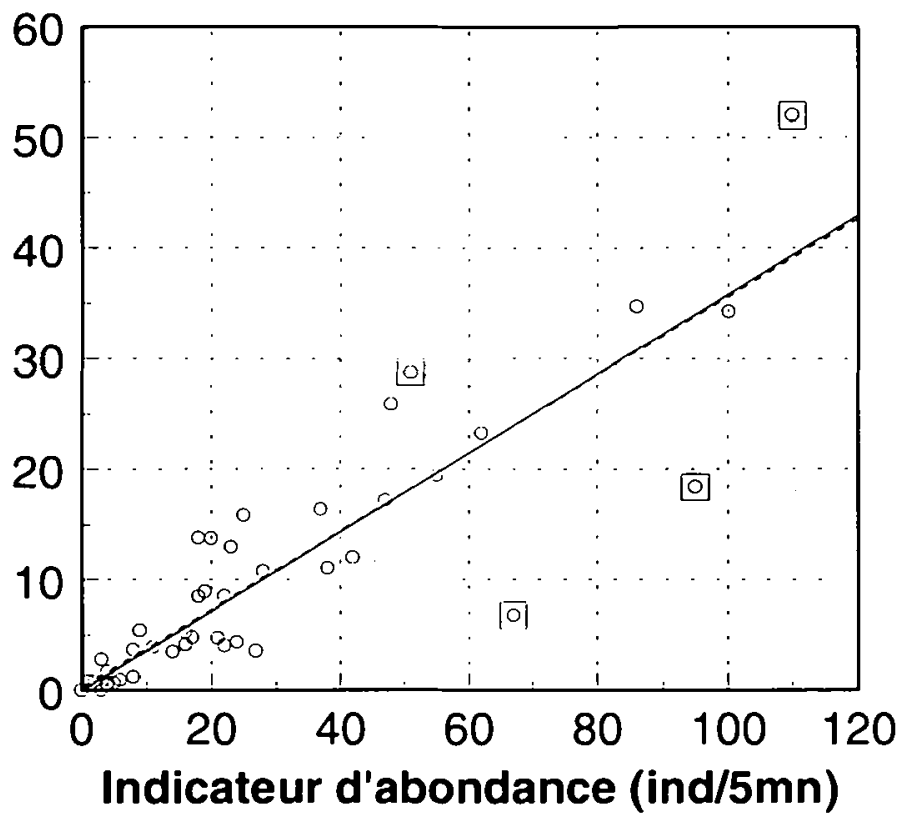

Droites de régression

$$
Y=a x+b
$$

$a=0,358 ; b=0,290$

S.C.E $=1209$

$R^{2}=0,78 ; R=0,88$

$$
\begin{gathered}
Y=a X \\
a=0,358 \\
\text { S.C.E. }=1211
\end{gathered}
$$

Figure 2

Relation entre les indicateurs d'abondance et les estimations de densité. Les quatre points présentant les plus forts résidus par rapport aux droites de régression sont encadrés.

\section{Figure 2}

Abundance indices vs density estimates relationship. The four points with the largest residuals from the regression lines are surrounded with a square.

\section{DISCUSSION}

La méthode bayesienne retenue pour procéder aux estimations de densité donne des estimations ponctuelles correspondant au maximum de vraisemblance, sous réserve que la vraisemblance conjointe de $N$ et $p$ possède un maximum à l'intérieur de la gamme des valeurs possibles fixée par les distributions a priori (cas général correspondant à des opérations de pêche électrique pratiquées avec un minimum de rigueur). Néanmoins, on a choisi de s'écarter des méthodes les plus couramment employées (COWX, 1983) et fondées sur le même critère du maximum de vraisemblance. Outre l'intérêt spécifique que l'on peut trouver dans une approche bayesienne, on a cherché à obtenir une vision plus réaliste de l'incertitude des estimations de densité, que ce soit par la variance des distributions de probabilité a posteriori ou par les intervalles a posteriori à $95 \%$. En effet, les méthodes d'estimation classiques proposent des estimateurs de variance asymptotiques qui sont fiables quand $N$ et $p$ sont grands (SEBER, 1982). Ces estimateurs asymptotiques peuvent sous-estimer (parfois grandement) les variances d'estimation pour des opérations de pêche électrique telles que pratiquées usuellement, c'est-à-dire sur des secteurs de quelques centaines de $\mathrm{m}^{2}$ avec, dans le cas des juvéniles de saumon atlantique, des densités le plus souvent inférieures à $15 \mathrm{ind} / 100 \mathrm{~m}^{2}$. Les intervalles de confiance dérivés de ces estimateurs de variance asymptotiques sont à leur tour eux aussi suspects dans beaucoup de cas rencontrés dans la pratique usuelle des pêches électriques. Les intervalles a posteriori à $95 \%$ tels que présentés à la figure 1 donnent certainement une vision plus réaliste, mais aussi plus pessimiste, du degré de confiance que l'on peut accorder aux estimations de densité déduites des opérations de captures successives. SCHNUTE (1983) propose également une approche allant dans le même sens que celle utilisée ici, sans pour autant adopter un point de vue bayesien. 
Toutes les données disponibles ont été utilisées, quelle que soit leur précision, pour caler la relation indicateur d'abondance/estimation de densité. En effet, on a choisi de procéder à l'analyse dans une configuration, en terme de qualité des données, la plus proche possible de celle rencontrée en règle générale dans les bases de données actuellement disponibles sur les abondances de juvéniles de saumon. La forte incertitude de certaines estimations de densité a été jugée comme reflétant la qualité variable des données récoltées couramment par la méthode des enlèvements successifs par pêche électrique. En outre, si l'on avait choisi de ne pas retenir certaines données, seuls les cas d'estimations de densité jugées trop incertaines auraient pu être écartés, alors que ceux d'indicateurs d'abondance douteux n'auraient pu être traités de la même façon, faute de disposer d'une mesure de précision pour ces derniers. Quoi qu'il en soit, des analyses complémentaires non rapportées dans cet article montrent que la relation indicateur d'abondance/estimation de densité présentée n'est pas sensiblement modifiée par le retrait des points associés à des estimations de densité peu précises ou présentant de forts résidus.

L'analyse des données montre qu'il existe, pour les juvéniles de saumon de l'année, une liaison claire entre indicateurs d'abondance et estimations de densité, que l'on peut aisément modéliser par une relation directe de proportionnalité. La raison fondamentale de l'existence d'une telle relation directe de proportionnalité réside certainement dans le protocole standardisé mis au point pour les indicateurs d'abondance (PRÉVOST et BAGLINIĖRE, 1995). En effet, on fait en sorte que chaque " coup d'électrode " soit donné de la même façon, ce qui combiné avec un temps de pêche fixe, doit conduire à prospecter une surface à peu près identique lors de chaque opération et à capturer une proportion relativement constante des individus présents sur la surface prospectée. Dans l'optique d'une analyse de la précision des indicateurs d'abondance de type CPUE, ce point crucial devra être examiné de façon critique.

Même si elle constitue également un élément de validation mutuelle des deux méthodes de quantification de l'abondance des tacons $0+$ automnaux, la relation présentée dans cet article est avant tout une relation de conversion d'un indicateur d'abondance de type CPUE en une densité, telle qu'estimée par des enlèvements successifs. La variabilité résiduelle autour de la relation moyenne entre indicateur d'abondance et estimation de densité reflète en partie le fait que les deux méthodes donnent des mesures de l'abondance réelle entachées d'une erreur aléatoire. On peut donc penser que la liaison entre indicateur d'abondance et abondance réelle est encore plus nette que celle mise en évidence avec les estimations de densité, avec cependant un coefficient de proportionnalité supérieur, car les densités estimées par enlèvements successifs sont connues pour être biaisées négativement (BOHLIN et al., 1990 ; RILEY et FAUSCH, 1992 ; RODGERS et al., 1992 ; RILEY et al., 1993).

Pour la relation présentée ici, la variabilité résiduelle est pour l'essentiel due à quatre couples de données (Figure 2, points encadrés) qui peuvent aisément être expliqués par des contraintes inhérentes à la collecte des données de terrain. En effet, les secteurs prospectés sur chaque station peuvent ne pas se recouvrir exactement et comprendre des zones de qualité différente suivant le type de données collectées, tout en restant dans la gamme des habitats courants, peu profonds et à substrat grossier globalement favorables aux tacons $0+$ automnaux. Les deux situations où l'indicateur d'abondance donne une vision optimiste de l'abondance des juvéniles de saumon par rapport aux estimations de densité correspondent à des stations où l'indicateur d'abondance a été obtenu en prospectant sélectivement les habitats les plus favorables aux juvéniles alors que les pêches par enlèvements successifs ont porté sur des secteurs d'un seul tenant mais comportant des zones d'habitat sub-optimal pour le saumon. Les deux cas où l'indicateur d'abondance donne une vision pessimiste de l'abondance des juvéniles par rapport aux estimations de densité correspondent à la situation inverse, 
à savoir que les pêches par enlèvements successifs ont porté sur une zone très homogène d'habitat optimal pour les tacons $0+$ mais de taille trop réduite pour permettre cinq minutes de pêche selon le protocole standardisé utilisé pour les indicateurs d'abondance. Ces derniers ont donc été collectés en incluant des habitats un peu moins favorables à la périphérie du secteur prospecté pour les estimations de densité. En dehors de ces problèmes de correspondance entre domaines d'échantillonnage suivant le type de données recueillies, aucune des variables complémentaires disponibles. (superficie et largeur de la station, écart entre les dates des deux types d'opérations, taille moyenne des individus capturés, écart-type des estimations de densité) n'a permis. d'expliquer :les résidus.

Si l'on s'en tient au protocole présenté par PRÉVOST et BAGLINIĖRE (1995), la densité estimée par enlèvements successifs exprimée en nombre d'individus par $100 \mathrm{~m}^{2}$ est en moyenne, pour un niveau d'indicateur d'abondance donné, de l'ordre d'un tiers du nombre de tacons capturés en cinq minutes. Cette relation a été ajustée pour des rivières de plus de $3 \mathrm{~m}$ de large et ne devrait pas être extrapolée pour des cours d'eau plus étroits. En effet, les quelques données disponibles pour des ruisseaux de moins de $3 \mathrm{~m}$ de large (huit couples [indicateur d'abondance ; estimation de densité]) suggèrent qu'il existe toujours une relation de proportionnalité directe entre les deux types de mesure d'abondance mais que le coefficient de proportionnalité n'est plus le même $(0,869$; intervalle de confiance bootstrap à $95 \%[0,681 ; 1,380]$; PRÉVOST et NIHOUARN, données non publiées). Ainsi, des indicateurs d'abondance collectés sur des cours d'eau de plus de $3 \mathrm{~m}$ de large ne devraient pas être comparés directement avec ceux obtenus sur des ruisseaux de largeur inférieure à $3 \mathrm{~m}$. Il en va de même si l'on tentait d'utiliser la relation présentée dans cet article dans des écosystèmes très différents de ceux des rivières du Massif Armoricain, tels que par exemple des cours d'eau de plus grande taille. Des réserves similaires peuvent être émises à l'encontre d'une extrapolation directe à d'autres stades que le juvénile de l'année ou d'autres espèces que le saumon atlantique (en particulier les stades plus âgés ou la truite commune (Salmo trutta) qui ont des preferundums d'habitats différents des tacons 0+ automnaux ; BAGLINIËRE et CHAMPIGNEULLE, 1982 ; HEGGENES et al., 1995). Les résultats présentés dans cet article permettent cependant de spéculer a priori que, pour les juvéniles de saumon $0+$ automnaux et dans des contextes écologiques relativement homogènes, un protocole du même type que celui proposé par PRÉVOST et BAGLINIĖRE (1995) permet d'obtenir simplement des indicateurs d'abondance proportionnels à des densités estimées par enlèvements successifs, même si la relation de conversion doit être établie au cas par cas.

\section{REMERCIEMENTS}

Les travaux présentés dans cet article ont fait l'objet d'une convention INRA/CSP $n^{\circ}$ 97594. Les auteurs tiennent à remercier les techniciens INRA et gardes-pêche du CSP qui ont participé à la collecte des données sur le terrain.

\section{BIBLIOGRAPHIE}

ANONYME, 1996a. SYSTAT 6.0 for Windows ${ }^{\oplus}$ : Statistics. SPSS Inc., Chicago, 751 p. ANONYME, 1996b. SYSTAT 6.0 for Windows ${ }^{\circ}$ : Graphics. SPSS Inc., Chicago, $450 \mathrm{p}$. BAGLINIĖRE J.L., CHAMPIGNEULLE A., 1982. Densité des populations de truite commune (Salmo trutta L.) et de juvéniles de saumon atlantique (Salmo salar L.) sur le cours principal du Scortt (Bretagne) : preferundums physiques et variations annuelles (1976 - 1980). Acta CEcologica CEcol. Appl., 3, 241-256. 
BAGLINIĖRE J.L., MAISSE G., NIHOUARN A., 1993. Comparison of two methods of estimating Atlantic salmon, Salmo salar, wild smolt production. In GIBSON R.J., CUTTING R.E. (eds), Production of juvenile Atlantic salmon, Salmo salar, in natural waters, Can. Spec. Publ. Fish. Aquat. Sci., 118, 189-201.

BOHLIN T., COWX I.G., 1990. Implications of unequal probability of capture by electric fishing on the estimation of population size. In COWX I.G. (ed.), Developments in electric fishing, 145-155, Fishing News Books, Oxford.

BOHLIN T., HEGGBERGET T.G., STRANGE C., 1990. Electric fishing for sampling and stock assessment. In COWX I.G., LAMARQUE P. (eds), Fishing with electricity. Applications in freshwater fisheries management, 112-139, Fishing News Books, Oxford.

CARLE F.L., STRUB M.R., 1978. A new method for estimating population size from removal data. Biometrics, 34, 621-630.

COBLE D.W., 1992. Predicting population density of largemouth bass from electrofishing catch per effort. North Am. J. Fish. Mgmt., 12, 650-652.

COWX I.G., 1983. Review of the methods for estimating fish population size from survey removal data. Fish. Mgmt., 14, 67-82.

CROZIER W.W., KENNEDY G.J.A., 1994. Application of semi-quantitative electrofishing to juvenile salmonid stock surveys. J. Fish. Biol., 45, 159-164.

EDWARDS C.M., DRENNER R.W., GALLO K.L., RIEGER K.E., 1997. Estimation of population density of largemouth bass in ponds by using mark-recapture and elctrofishing catch per effort. North Am. J. Fish. Mgmt., 17, 719-725.

HANKIN D.G., 1984. Multistage sampling designs in fisheries research : applications in small streams. Can. J. Fish. Aquat. Sci. 41, 1575-1591.

HEGGENES J., BAGLINIĖRE J.L., CUNJAK R., 1995. Note de synthèse sur la sélection de niche spatiale et la compétition chez le jeune saumon atlantique (Salmo salar) et la truite commune (Salmo trutta) en milieu lotique. Bull. Fr. Pêche Piscic., 337/338/339, 231-239.

JONES M.L., STOCKWELL J.D, 1995. A rapid assessment procedure for the enumeration of salmonine populations in streams. North Am. J. Fish. Mgmt., 15, 551-562.

LOBÓN-CERVIÁ J., UTRILLA C.G., 1993. A simple model to determine stream trout (Salmo trutta L.) densities based on one removal with electrofishing. Fish. Res., 15, 369-378.

MORAN P.A.P., 1951. A mathematical theory of animal trapping. Biometrika, 38, 307-311.

NELVA A., PERSAT H, CHESSEL D., 1979. Une nouvelle méthode d'étude des peuplements ichtyologiques dans les grands cours d'eau par échantillonnage ponctuel d'abondance. C.R. acad. sci. Paris, 289(D), 1295-1298.

PRÉVOST E., BAGLINIĖRE J.L., 1995. Présentation et premiers éléments de mise au point d'une méthode simple d'évaluation du recrutement en juvéniles de saumon atlantique (Salmo salar) de l'année en eau courante. In GASCUEL D., DURAND J.L., FONTENEAU A. (eds), Les recherches françaises en évaluation quantitative et modélisation des ressources halieutiques, Actes du colloque,

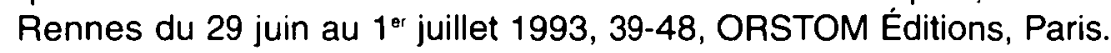

RILEY S.C., FAUSCH, K.D., 1992. Underestimation of trout population size by maximum-likelihood removal estimates in small streams. North Am. J. Fish. Mgmt., 12, 768-776.

RILEY S.C., HAEDRICH R.L., GIBSON R.J., 1993. Negative bias in removal estimates of Atlantic salmon parr relative to stream size. J. Freshw. Ecol., 8, 97-101.

RODGERS J.D., SOLAZZI M.F., JONSON S.L., BUCKMAN M. A., 1992. Comparison of three techniques to estimate juvenile coho salmon populations in small streams. North Am. J. Fish. Mgmt., 12, 79-86.

SCHNUTE J., 1983. A new approach to estimating populations by the removal method. Can. J. Fish. Aquat. Sci., 40, 2153-2169. 
SEBER G.A.F., 1982. The estimation of animal abundance and related parameters. Charles Griffin and Company Ltd, Londres, $654 \mathrm{p}$.

SIMONSON T.D., LYONS J., 1995. Comparison of catch per effort and removal procedures for sampling stream fish assemblages. North Am. J. Fish. Mgmt., 15, 419-427.

STRANGE C.D.; APRAHAMIAN M.W., WINSTONE A.J., 1989. Assessment of a semi-quantitative electric fishing sampling for juvenile. Atlantic salmon, Salmo salar L., and trout, Salmo trutta L., in small streams. Aquacult. Fish. Mgmt., $20,485-492$. 